Research Article

\title{
Comparison of Different Phenotypic Tests versus PCR in the Detection of Carbapenemase-Producing Pseudomonas aeruginosa Isolates in Hamadan, Iran
}

\author{
Masoumeh Beig $\mathbb{D},{ }^{1}$ Mohammad Taheri $\mathbb{D},{ }^{1}$ and Mohammad Reza Arabestani $\mathbb{B D}^{1,2}$ \\ ${ }^{1}$ Department of Microbiology, Hamadan University of Medical Sciences, Hamadan, Iran \\ ${ }^{2}$ Nutrition Health Research Center, School of Medicine, Hamadan University of Medical Sciences, Hamadan, Iran \\ Correspondence should be addressed to Mohammad Reza Arabestani; mohammad.arabestani@gmail.com
}

Received 25 February 2021; Revised 23 April 2021; Accepted 8 July 2021; Published 19 July 2021

Academic Editor: Chioma Blaise Chikere

Copyright (c) 2021 Masoumeh Beig et al. This is an open access article distributed under the Creative Commons Attribution License, which permits unrestricted use, distribution, and reproduction in any medium, provided the original work is properly cited.

\begin{abstract}
In recent years, the prevalence of carbapenem-resistant Pseudomonas aeruginosa isolates has become a worldwide concern. Rapid and accurate detection of carbapenemase-producing $P$. aeruginosa isolates is so important. The aim of this study was to evaluate the performance of the phenotypic methods such as Modified Hodge test (MHT), CarbaNP (CNPt), combined double-disk synergy test (CDDT), and carbapenem inactivation method (CIM) for rapid and accurate detection of clinical carbapenemase production of $P$. aeruginosa isolates. This study was performed on $97 \mathrm{P}$. aeruginosa strains, which were isolated from clinical samples in Hamadan hospitals, western Iran in 2017-2018. Antibiotic susceptibility testing was performed using disk diffusion and minimum inhibitory concentration (MIC) by E-test method. We evaluated the performance of MHT, CarbaNP, CDDT, and CIM tests in comparison to polymerase chain reaction (PCR) for the detection of carbapenemase-producing isolates. Additionally, the presence of carbapenem-resistant genes was investigated using the PCR method. Our findings showed that the highest resistance was to cefoxitin (94.8\%). Moreover, among the carbapenem antibiotics, the highest resistance was to imipenem (49.4\%). Among the 49 carbapenem-resistant isolates, $42(85.7 \%)$ isolates were MIC positive. The results of phenotypic tests showed that CarbaNP, CIM, CDDT, and MHT tests were positive in (48/49, 97.95\%), (46/49, 93.87\%), (27/49, 57.44\%), and (25/49, 53.19\%) of isolates, respectively. CarbaNP and CIM tests showed high sensitivity, specificity, positive predictive values (PPV), and negative predictive values (NPV) compared to PCR in P. aeruginosa isolates. CarbaNP and CIM tests are highly sensitive and specific tests for identifying carbapenemase-producing $P$. aeruginosa isolates.
\end{abstract}

\section{Introduction}

Pseudomonas aeruginosa is an opportunistic pathogen and is a major cause of nosocomial infections worldwide [1]. However, carbapenems are considered the last line in the treatment of severe infections caused by $P$. aeruginosa isolates [2]. Over the past decade, the emergence of carbapenem-resistant isolates has become a major concern in health care systems. Although multiple mechanisms of carbapenem resistance have been reported, most of the mechanisms are relevant to the prevalence of carbapenemases enzymes, belonging to Ambler class A (KPCs), class B (VIMs, IMP, SPM, SIM, and GIM), and class D (OXA-48) $\beta$-lactamases [3]. Bacterial isolates, which are capable of producing carbapenemase enzymes, have the ability to inactivate a wide range of $\beta$-lactams, including penicillins, cephalosporins, carbapenems, and monobactams [4].

These isolates can spread rapidly in the hospital environment causing nosocomial infections with high mortality. So, rapid identification of carbapenemase-producing $P$. aeruginosa isolates is most important for timely detection, treatment, and performance of infection control measures to prevent the expansion of these resistant isolates [5]. Although molecular methods remain the gold standard for the identification of carbapenemase-producing isolates and enzyme types, carbapenemase genes can be easily detected 
by PCR [6]. However, for reasons such as the high cost and the inability of this method to detect new carbapenemase genes, various phenotypic methods such as CarbaNP, CIM, MHT, and CDDT have been developed for the rapid detection of carbapenemase-producing isolates [6]. In our study, we investigated the diagnostic values of four phenotypic tests (CarbaNP, CIM, MHT, and CDDT) for the detection of carbapenemase-producing $P$. aeruginosa isolates. CarbaNP and CIM tests are two of the phenotypic tests, which can be done with routine laboratory equipment (in-house) and have been recently recommended by Clinical and Laboratory Standards Institute (CLSI) guidelines for the detection of carbapenemase-producing P. aeruginosa isolates [7].

\section{Materials and Methods}

2.1. Isolation and Identification of $P$. aeruginosa. This descriptive cross-sectional study was carried out through November 2017 to May 2018. A total of 97 P. aeruginosa isolates were collected from hospitalized patients via different clinical specimens including urine, wound, blood, trachea, and other clinical specimens. They were hospitalized in the educational hospitals of Hamadan University of Medical Sciences, Hamadan, Iran. Initially, after the collected samples were transferred to the microbiological laboratory, they were cultured on a blood agar medium and the pure colonies were isolated. Then, Gram staining and various biochemical tests, including oxidase, catalase, oxidative-fermentative test, growth on media including triple sugar iron agar (TSI), Cetrimide agar, and growth at $42^{\circ} \mathrm{C}$, were performed to identify $P$. aeruginosa isolates. Finally, the isolates were kept in brain heart infusion (BHI) media containing $20 \%$ glycerol and stored at $-70^{\circ} \mathrm{C}$ [8]. This study was approved by the ethics committee of the University of Medical Sciences, Hamadan, Iran (Code No.: IR.UMSHA.REC.1396.662).

2.2. Antimicrobial Susceptibility Testing. Antimicrobial susceptibility testing for $P$. aeruginosa isolates was performed according to the CLSI (2017) instructions using the disk diffusion method (Kirby-Bauer) on Mueller-Hinton agar plates to various antibiotics [9]. All disks, including imipenem $(10 \mu \mathrm{g})$, meropenem $(10 \mu \mathrm{g})$, doripenem $(10 \mu \mathrm{g})$, ceftazidime $(30 \mu \mathrm{g})$, amikacin $(30 \mu \mathrm{g})$, tetracycline $(75 \mu \mathrm{g})$, piperacillin/tazobactam $(1000 / 10 \mu \mathrm{g})$, piperacillin $(100 \mu \mathrm{g})$, ceftriaxone $(30 \mu \mathrm{g})$, cefotaxime $(30 \mu \mathrm{g})$, cefoxitin $(30 \mu \mathrm{g})$, and ciprofloxacin $(5 \mu \mathrm{g})$, were purchased from the British MAST Group (UK). For samples resistant to carbapenem, the MIC test was performed by the imipenem E-test. P. aeruginosa ATTC27853 was used as a standard strain.

2.3. Selection of P. aeruginosa Isolates for Phenotypic and PCR Tests. Clinical isolates based on carbapenem resistance profile were selected and examined for phenotypic and PCR tests.

2.4. Phenotypic Detection of Metallo- $\beta$-lactamase (MBL) by Combined Double-Disk Synergy Test (CDDT). CDDT was used for phenotypic identification of MBLs producing $P$. aeruginosa isolates. Imipenem (IMP) $(10 \mu \mathrm{g})$ and imipenem + ethylenediaminetetraacetic acid (EDTA) discs were used to detect MBL-producing $P$. aeruginosa isolates. After $18-24$ hours of incubation at $35^{\circ} \mathrm{C}$, if the increase in inhibition zone with the imipenem-EDTA disk was $\geq 7 \mathrm{~mm}$ compared to the IMP disk alone, it was considered as MBL positive [10].

2.5. Modified Hodge Test (MHT). This phenotypic test is recommended by CLSI (2017) to detect carbapenemaseproducing bacteria. At first, a $0.5 \mathrm{McF}$ arland dilution of Escherichia coli (ATCC 25922) in $5 \mathrm{ml}$ of broth or saline was prepared. A 1:10 dilution was streaked as lawn on to a Mueller Hinton agar (MHA) plate. A $10 \mu \mathrm{g}$ ertapenem disk (Mast, UK) was placed on the center of the plate [11]. $P$. aeruginosa isolates (test isolates) were streaked in four different directions. $P$. aeruginosa isolates were cultured in a direct line from the edge of the disc outwards to the periphery of the plate. The plates were then incubated at $37^{\circ} \mathrm{C}$ for 16 to 24 hours. The cloverleaf-like structure indicated the production of carbapenemase by the test isolates. Klebsiella pneumoniae (ATCC BAA-1705) was used as a positive control [12].

2.6. Carbapenem Inactivation Method (CIM). In this test, for each one of the isolates, a $10 \mu \mathrm{L}$ loop of culture was suspended in a $2 \mathrm{~mL}$ tryptic soy broth (TSB) medium. A meropenem disk was added to each tube using a sterile loop. After the incubation of the tubes for 2 hours at $35^{\circ} \mathrm{C} \pm 2^{\circ} \mathrm{C}$ [13], meropenem disk was removed from the suspension, then placed on Mueller-Hinton agar plate, inoculated with a susceptible E. coli indicator strain (ATCC 29522), and subsequently incubated at $35^{\circ} \mathrm{C}$ for $18-24$ hours. When the suspected isolates produce the carbapenemase enzymes, the meropenem disc is inactivated and the susceptible indicator strain can grow in the presence of the inactive disc [14].

2.7. CarbaNP Test (CNPt). First, two microcentrifuge tubes were labeled as (a) and (b); then, $100 \mu \mathrm{l}$ of the bacterial reagent was added to each tube. For each isolate, $1 \mu \mathrm{L}$ of bacteria from an overnight blood agar plate in both tubes was inoculated. Then, $100 \mu \mathrm{l}$ of solutions A and B was added to tubes "a" and "b," respectively, and they were vortexed. The tubes were then incubated at $35^{\circ} \mathrm{C}$ for up to 2 hours [12]. After 2 hours, the carbapenemase-producing isolates cause the $\mathrm{pH}$ shift and produced yellow color, but the isolates that do not produce carbapenemase enzymes remained the same color as the solution [15].

2.8. DNA Extraction. Total DNAs of P. aeruginosa isolates were extracted by the boiling method. Briefly, 3-5 colonies of overnight bacterial culture were suspended again in $500 \mu \mathrm{l}$ of sterile distilled water, boiled for $30 \mathrm{mins}$, and then centrifuged at $14000 \mathrm{~g}$ for $5 \mathrm{mins}$ to pellet cell debris. Then, the extracted DNA was stored at $-20^{\circ} \mathrm{C}[16,17]$. The quantity and quality of the DNA were determined using a nano 
spectrophotometer (NanoDrop ND-1000, Biocompare, San Francisco, USA) and gel electrophoresis.

2.9. Detection of Carbapenemase-Encoding Genes by PCR. Carbapenem-resistant $P$. aeruginosa isolates were tested for KPC, IMP, VIM, SIM, GIM, SPM, OXA-48, and AMPC genes by PCR using specific primers (Table 1) [18-21].

2.10. Statistical Analysis. SPSS 19 was incorporated in the analysis of the collected data (Chicago, IL, USA). Student's $t$ test was used to analyze numerical data. The statistical significance was $P<0.05$. The sensitivity and specificity of phenotypic methods were analyzed against PCR as a goldstandard method by the Chi-square test.

\section{Results}

3.1. Antimicrobial Susceptibility Testing of P. aeruginosa Isolates. The results of antimicrobial susceptibility test on 97 $P$. aeruginosa isolates showed that the highest resistance was to cefoxitin $(94.8 \%, n=92)$ and the lowest resistance was to piperacillin/tazobactam $(39.2 \%, n=38)$. Moreover, among the carbapenem antibiotics, the highest resistance was to imipenem $(49.4 \%, n=48)$ and the lowest resistance was to meropenem $(41.2 \%, n=40)$. Furthermore, among the 97 $P$. aeruginosa isolates, 49 isolates were resistant and 48 isolates were susceptible to carbapenems (Table 2). Out of 49 (51.51\%) carbapenem-resistant isolates, 42 (85.7\%) isolates had positive results for MIC (Table 3).

3.2. Demographic Characteristics of Evaluated P. aeruginosa Isolates and Antibiogram Resistance. Association of the demographic characteristics of evaluated $P$. aeruginosa isolates and antibiogram resistance has been shown in Table 4.

3.3. Results of Isolates Containing Different Classes of Carbapenemase. Out of 49 carbapenems-resistant $P$. aeruginosa isolates which were divided into different Ambler groups including KPC (11/49), B (40/49), AmpC (25/49), and OXA-48 (35/49) and while 48 isolates were susceptible to this antibiotic family, these 49 carbapenemresistant isolates were tested for different phenotypic and PCR tests.

3.4. Phenotypic Tests for the Detection of Class A BetaLactamase. The highest sensitivity and specificity of phenotypic tests for the detection of KPC gene were related to CarbaNP and CIM tests (100\%), although MHT and CDDT tests had lower sensitivity and specificity for the detection of KPC gene (Tables 5 and 6).

3.5. Phenotypic Tests for the Detection of Class B BetaLactamase. The highest sensitivity and specificity of phenotypic tests for the detection of IMP, VIM, SIM, SPM, and
GIM genes were related to CarbaNP and CIM tests (100\%) (Tables 5 and 6).

3.6. Phenotypic Tests for the Detection of Class C BetaLactamase. The highest sensitivity and specificity of phenotypic tests for the detection of $A m p C$ gene were related to CarbaNP tests (100\%) (Tables 5 and 6).

3.7. Phenotypic Tests for the Detection of Class D BetaLactamase. The highest sensitivity and specificity of phenotypic tests for the detection of OXA-48 gene were related to CarbaNP tests ( $97 \%$ and $100 \%$, respectively) (Tables 5 and 6).

3.8. Detection of Carbapenemase-Encoding Genes by PCR. The results of the PCR method showed that 11 isolates were producer class A (KPC), 40 isolates class B (IMP, VIM, SIM, SPM, and GIM), 25 isolates class $\mathrm{C}$ (AmpC), and 35 isolates class D (OXA-48) carbapenemases.

3.9. Phenotypic Tests for the Detection of Combination of Classes A, B, C, and D Beta-Lactamases. The performances of four different phenotypic tests were variable for the 35 $P$. aeruginosa isolates carrying the class $\mathrm{D}$ carbapenemase (OXA-48). Generally, the highest positive detection was related to CarbaNP (34/35, 97\%), with one missing OXA-48producing isolate as well as one strain harboring both the OXA-48 and AmpC genes. Out of the 35 P. aeruginosa isolates carrying the class D carbapenemase (OXA-48), 20 isolates (70\%) were detected by CDDT test; also out of 25 isolates carrying $\mathrm{AmpC}$ cephalosporinase gene, 16 isolates (73\%) were detected by CDDT test, and this test could not detect 15 isolates carrying OXA-48 gene and 9 isolates carrying AmpC gene. In this regard, the MHT test had the most unpleasant result; it detected only 18 isolates out of the 35 isolates carrying OXA-48 gene (67\%) and 14 isolates out of the 25 isolates carrying $\mathrm{AmpC}$ cephalosporinase gene (69\%) and missed detecting 11 isolates carrying AmpC gene. Out of 49 carbapenemase-producing isolates, 5 isolates had VIM plus KPC genes simultaneously. These isolates were successfully identified by CarbaNP and CIM tests (5/5, $100 \%)$, but these isolates can be detected by the MHT test (4/ $5,80 \%)$ and CDDT test $(3 / 5,60 \%)$ (Tables 5 and 6).

The performance of the phenotypic methods is listed in Table 3. The CarbaNP and CIM tests leading to comparable sensitivities ( $97 \%$ vs. $94 \%, P=0.02)$, were higher than that of the MHT and CDDT tests $(P<0.003)$. Owing to the improved detection of the OXA-48 gene carrier isolates, the sensitivity of the CarbaNP test was significantly higher than that of the MHT and CDDT tests (97\% vs. $67 \%$ and $70 \%$, $P<0.001)$. Similarly, the sensitivity of the CarbaNP and CIM tests, increased to $96 \%$ and $89 \%$, which was attributed to the improvement in the detection of the AmpC gene carrier isolates. Specificity of the CarbaNP, CDDT, MHT and CIM tests for the detection of AmpC gene were $100 \%, 100 \%$, 88\%, 96\% specificity. Taken together, the CarbaNP and CIM tests possessed the best performance for the efficient detection of 
TABLE 1: PCR primers for the detection of carbapenemase genes.

\begin{tabular}{|c|c|c|c|c|}
\hline Carbapenemase genes & Sequence $\left(5^{\prime}-3^{\prime}\right)$ & Primer & Expected amplicon size (bp) & Reference \\
\hline IMP & $\begin{array}{l}\text { Imp-F } \\
\text { Imp-R }\end{array}$ & $\begin{array}{c}\text { GGA ATA GAG TGG CTT AAY TCT C } \\
\text { CCA AAC YAC TAS GTT ATC T }\end{array}$ & 188 & {$[18]$} \\
\hline VIM & $\begin{array}{l}\text { Vim-F } \\
\text { Vim-R }\end{array}$ & $\begin{array}{l}\text { GAT GGT GTT TGG TCG CAT A } \\
\text { CGA ATG CGC AGC ACC AG }\end{array}$ & 390 & {$[18]$} \\
\hline GIM-1 & $\begin{array}{l}\text { GIM-1F } \\
\text { GIM-2R }\end{array}$ & $\begin{array}{l}\text { TCG ACA CAC CTT GGT CTG AA } \\
\text { AAC TTC CAA CTT TGC CAT GC }\end{array}$ & 271 & {$[18]$} \\
\hline SPM-1 & $\begin{array}{l}\text { SPM-1F } \\
\text { SPM-1R }\end{array}$ & $\begin{array}{l}\text { AAA ATC TGG GTA CGC AAA CG } \\
\text { ACA TTA TCC GCT GGA ACA GG }\end{array}$ & 477 & {$[18]$} \\
\hline SIM-1 & $\begin{array}{l}\text { Sim-1F } \\
\text { Sim-1R }\end{array}$ & $\begin{array}{l}\text { TAC AAG GGA TTC GGC ATC G } \\
\text { TAA TGG CCT GTT CCC ATG TG }\end{array}$ & 570 & {$[18]$} \\
\hline KPC $1-5$ & $\begin{array}{l}\mathrm{KPC}-1 \mathrm{~F} \\
\mathrm{KPC}-1 \mathrm{R}\end{array}$ & $\begin{array}{c}\text { CATTCAAGGGCTTTCTTGCTGC } \\
\text { ACGACGGCATAGTCATTTGC }\end{array}$ & 538 & {$[19]$} \\
\hline AMPC & $\begin{array}{l}\text { AMPC-F } \\
\text { AMPC-R }\end{array}$ & $\begin{array}{l}\text { CGGCTCGGTGAGCAAGACCTTC } \\
\text { AGTCGCGGATCTGTGCCTGGTC }\end{array}$ & 218 & {$[20]$} \\
\hline OXA-48 & $\begin{array}{l}\text { OXA-48-F } \\
\text { OXA-48-R }\end{array}$ & $\begin{array}{c}\text { GCTTGATCGCCCTCGATT } \\
\text { GATTTGCTCCGTGGCCGAAA }\end{array}$ & 281 & {$[21]$} \\
\hline
\end{tabular}

TABLE 2: The results of antibiogram testing for P. aeruginosa isolate.

\begin{tabular}{|c|c|c|c|c|}
\hline Antibiotic & No. resistant (\%) & Intermediate & Sensitive & Total \\
\hline Piperacillin & $42(43.3)$ & $13(13.4)$ & $42(43.3)$ & 97 \\
\hline Piperacillin/tazobactam & $38(39.2)$ & $11(11.3)$ & $48(49.4)$ & 97 \\
\hline Ceftazidime & $40(41.2)$ & $6(6.2)$ & $51(52.6)$ & 97 \\
\hline Aztreonam & $50(51.5)$ & $19(19.5)$ & $28(28.9)$ & 97 \\
\hline Amikacin & $40(41.2)$ & $10(10.3)$ & $47(48.5)$ & 97 \\
\hline Ciprofloxacin & $53(54.6)$ & $2(2.1)$ & $42(43.3)$ & 97 \\
\hline Meropenem & $40(41.2)$ & $5(5.2)$ & $52(53.6)$ & 97 \\
\hline Doripenem & $45(46.4)$ & $2(2.1)$ & $50(51.5)$ & 97 \\
\hline Cefoxitin & $92(94.8)$ & $1(1)$ & $4(4.1)$ & 97 \\
\hline Tetracycline & $53(54.6)$ & $5(5.2)$ & $39(40.2)$ & 97 \\
\hline Ceftriaxone & $65(67)$ & $18(18.6)$ & $14(14.43)$ & 97 \\
\hline Imipenem & $48(49.4)$ & $1(1)$ & $48(49.4)$ & 97 \\
\hline
\end{tabular}

TABLE 3: Results of MIC and different phenotypic tests for carbapenemase genes.

\begin{tabular}{|c|c|c|c|c|c|}
\hline Gene related to carbapenem resistance & MIC imipenem & CDDT & MHT & CIM & CarbaNP \\
\hline KPC & $>32$ & $6 / 11$ & $5 / 11$ & $11 / 11$ & $11 / 11$ \\
\hline IMP & 8 to $>32$ & $13 / 20$ & $15 / 20$ & $20 / 20$ & $20 / 20$ \\
\hline VIM & 8 to $>32$ & $10 / 19$ & $14 / 19$ & $19 / 19$ & $19 / 19$ \\
\hline AMPC & 8 to $>32$ & $16 / 25$ & $14 / 25$ & $22 / 25$ & $24 / 25$ \\
\hline OXA-48 & 8 to $>32$ & $20 / 35$ & $18 / 35$ & $33 / 35$ & $34 / 35$ \\
\hline SIM & 8 to $>32$ & $3 / 8$ & $4 / 8$ & $8 / 8$ & $8 / 8$ \\
\hline SPM & 16 & $10 / 17$ & $11 / 17$ & $17 / 17$ & $17 / 17$ \\
\hline GIM & 8 & $3 / 6$ & $3 / 6$ & $6 / 6$ & $6 / 6$ \\
\hline KPC + IMP + AMPC & 8 to $>32$ & $2 / 2$ & $2 / 2$ & $2 / 2$ & $2 / 2$ \\
\hline $\mathrm{KPC}+\mathrm{AMPC}+\mathrm{OXA}-48$ & 16 & $2 / 3$ & $1 / 3$ & $3 / 3$ & $3 / 3$ \\
\hline IMP + VIM + OXA-48 & 8 & $3 / 6$ & $6 / 6$ & $6 / 6$ & $6 / 6$ \\
\hline $\mathrm{IMP}+\mathrm{AMPC}+\mathrm{OXA}-48$ & $>32$ & $3 / 5$ & $4 / 5$ & $4 / 5$ & $5 / 5$ \\
\hline $\mathrm{IMP}+\mathrm{KPC}+\mathrm{OXA}-48$ & $>32$ & $1 / 1$ & $1 / 1$ & $1 / 1$ & $1 / 1$ \\
\hline $\mathrm{VIM}+\mathrm{KPC}+\mathrm{AMPC}$ & $>32$ & $1 / 3$ & $3 / 3$ & $3 / 3$ & $3 / 3$ \\
\hline $\mathrm{VIM}+\mathrm{KPC}+\mathrm{AMPC}+\mathrm{OXA}-48$ & 8 & $0 / 1$ & $1 / 1$ & $1 / 1$ & $1 / 1$ \\
\hline IMP + VIM + AMPC & $4-8$ & $2 / 4$ & $4 / 4$ & $4 / 4$ & $4 / 4$ \\
\hline $\mathrm{KPC}+\mathrm{OXA}-48+\mathrm{VIM}+\mathrm{AMPC}$ & $>32$ & $1 / 1$ & $1 / 1$ & $1 / 1$ & $1 / 1$ \\
\hline $\mathrm{IMP}+\mathrm{AMPC}$ & 8 & $8 / 11$ & $8 / 11$ & $11 / 11$ & $11 / 11$ \\
\hline VIM + KPC & 16 & $3 / 5$ & $4 / 5$ & $5 / 5$ & $5 / 5$ \\
\hline Not detected & $<0.25-2$ & $44 / 48$ & $45 / 48$ & $48 / 48$ & $48 / 48$ \\
\hline
\end{tabular}


TABle 4: Demographic characteristics of evaluated $P$. aeruginosa isolates and antibiogram resistance with three main antibiotics.

\begin{tabular}{|c|c|c|c|c|c|c|c|}
\hline \multirow{2}{*}{ Characteristics } & \multirow{2}{*}{ No. examined } & \multicolumn{2}{|c|}{ Imipenem resistance } & \multicolumn{2}{|c|}{ Meropenem resistance } & \multicolumn{2}{|c|}{ Doripenem resistance } \\
\hline & & No. (\%) & $P$-value & No. $(\%)$ & $P$-value & No. (\%) & $P$-value \\
\hline \multicolumn{8}{|l|}{ Age } \\
\hline $0-10$ & 9 & $5(55.6)$ & 0.308 & $5(55.6)$ & 0.441 & $5(55.6)$ & 0.346 \\
\hline $11-20$ & 9 & $5(55.6)$ & & $5(55.6)$ & & $5(55.6)$ & \\
\hline $21-30$ & 7 & $4(57.1)$ & & $3(42.8)$ & & $3(42.8)$ & \\
\hline $31-40$ & 7 & $3(42.8)$ & & $2(28.6)$ & & $2(28.6)$ & \\
\hline $41-50$ & 21 & $9(42.8)$ & & $9(42.8)$ & & $9(42.8)$ & \\
\hline $51-60$ & 14 & $5(35.7)$ & & $4(28.6)$ & & $3(21.4)$ & \\
\hline $61-70$ & 19 & $13(68.4)$ & & $12(63.1)$ & & $13(68.4)$ & \\
\hline $71-80$ & 5 & $2(40)$ & & $2(40)$ & & $3(60)$ & \\
\hline $81-90$ & 5 & $2(40)$ & & $2(40)$ & & $3(60)$ & \\
\hline $91-100$ & 1 & $1(100)$ & & $1(100)$ & & $1(100)$ & \\
\hline Total no. & 97 & $49(50.5)$ & & $45(46.4)$ & & $47(48.5)$ & \\
\hline \multicolumn{8}{|l|}{ Gender } \\
\hline Male & 70 & $34(48.6)$ & 0.232 & $33(47.1)$ & 0.488 & $34(48.6)$ & 0.345 \\
\hline Female & 27 & $15(55.6)$ & & $12(44.4)$ & & $13(48.1)$ & \\
\hline Total no. & 97 & $49(50.5)$ & & $45(46.4)$ & & $47(48.5)$ & \\
\hline \multicolumn{8}{|l|}{ Hospital ward } \\
\hline Burn & 28 & $19(67.8)$ & 0.223 & $19(67.8)$ & 0.170 & $18(64.3)$ & 0.154 \\
\hline Surgery & 5 & $2(40)$ & & $1(20)$ & & $0(0)$ & \\
\hline Pulmonary & 11 & $5(45.4)$ & & $4(36.4)$ & & $3(27.3)$ & \\
\hline Neurology & 3 & $0(0)$ & & $1(33.3)$ & & $1(33.3)$ & \\
\hline Orthopedics & 1 & $0(0)$ & & $0(0)$ & & $1(100)$ & \\
\hline Trauma & 13 & $7(53.8)$ & & $5(38.4)$ & & $6(46.1)$ & \\
\hline NICU & 8 & $2(25)$ & & $1(12.5)$ & & $1(12.5)$ & \\
\hline ICU & 14 & $9(64.3)$ & & $8(57.1)$ & & $10(71.4)$ & \\
\hline Hematology & 2 & $1(50)$ & & $0(0)$ & & $1(50)$ & \\
\hline Infected & 12 & $4(33.3)$ & & $6(50)$ & & $6(50)$ & \\
\hline Total no. & 97 & $49(50.5)$ & & $45(46.4)$ & & $47(48.5)$ & \\
\hline \multicolumn{8}{|l|}{ Sample source } \\
\hline $\mathrm{BC}$ & 22 & $14(63.6)$ & & $12(54.5)$ & & $12(54.5)$ & \\
\hline $\mathrm{UC}$ & 12 & $3(25)$ & 0.082 & $4(33.3)$ & 0.401 & $4(33.3)$ & 0.243 \\
\hline TC & 33 & $16(48.5)$ & & $14(42.4)$ & & $15(45.4)$ & \\
\hline TA & 5 & $3(60)$ & & $3(60)$ & & $4(80)$ & \\
\hline Wound & 15 & $9(60)$ & & $7(46.7)$ & & $8(53.3)$ & \\
\hline Sputum & 6 & $2(33.3)$ & & $3(50)$ & & $2(33.3)$ & \\
\hline CSF & 2 & $0(0)$ & & $0(0)$ & & $0(0)$ & \\
\hline Fluid & 1 & $1(100)$ & & $1(100)$ & & $1(100)$ & \\
\hline $\mathrm{TT}$ & 1 & $1(100)$ & & $1(100)$ & & $1(100)$ & \\
\hline Total no. & 97 & $49(50.5)$ & & $45(46.4)$ & & $47(48.5)$ & \\
\hline \multicolumn{8}{|c|}{ Hospitalization period } \\
\hline $0-10$ days & 56 & $20(35.7)$ & & $18(32.1)$ & & $14(25)$ & \\
\hline $11-29$ days & 25 & $16(64)$ & & $13(52)$ & & $18(72)$ & \\
\hline $1-2$ months & 16 & $13(81.2)$ & 0.433 & $14(87.5)$ & 0.455 & $15(93.7)$ & 0.601 \\
\hline Total no. & 97 & $49(50.5)$ & & $45(46.4)$ & & $47(48.5)$ & \\
\hline
\end{tabular}

Carbapenemase-producing isolates, among the four evaluated methods.

3.10. Results of Statistical Analysis. In P. aeruginosa isolates, there were significant correlations between the disk diffusion method and phenotypic results for carbapenem antibiotics $(P \leq 0.001)$ and between PCR and phenotypic results $(P \leq 0.001)$.

\section{Discussion}

Increased antibiotic resistance to carbapenems among $P$. aeruginosa isolates has become a public health problem.
The accurate and rapid detection of carbapenemase producing of $P$. aeruginosa isolates is necessary for appropriate treatment, prevention of spreading, and control of infections. In the last decade, phenotypic methods were extensively used in clinical laboratories for a first-line detection of the isolates producing carbapenemases [22]. These phenotypic methods have different sensitivity and specificity for the detection of carbapenemase-producing $P$. aeruginosa isolates. In the present study, among $97 P$. aeruginosa isolates, 49 isolates were identified as carbapenemase producers and 48 isolates were noncarbapenemase producers. Four phenotypic methods including MHT, CDDT, CarbaNP, and CIM tests were performed for the detection of 
TABLE 5: Results of sensitivity, specificity, NPV, PPV in CarbaNP versus CIM test.

\begin{tabular}{|c|c|c|c|c|c|c|c|c|c|c|c|}
\hline & \multirow{2}{*}{ No. } & \multicolumn{4}{|c|}{ CarbaNP } & \multirow{2}{*}{ No. } & \multicolumn{4}{|c|}{ CIM } & \multirow{2}{*}{ MIC } \\
\hline & & Sensitivity (\%) & Specificity (\%) & PPV* $\left.^{*} \%\right)$ & $\mathrm{NPV}^{*}(\%)$ & & Sensitivity (\%) & Specificity (\%) & PPV (\%) & NPV (\%) & \\
\hline A & $\begin{array}{c}11 / \\
11\end{array}$ & 100 & 100 & 100 & 100 & $\begin{array}{l}11 / \\
11\end{array}$ & 100 & 100 & 100 & 100 & $>32$ \\
\hline B & $\begin{array}{c}40 / \\
40\end{array}$ & 100 & 100 & 100 & 100 & $\begin{array}{c}39 / \\
40\end{array}$ & 97 & 100 & 100 & 88 & 8 to. 32 \\
\hline $\mathrm{C}$ & $\begin{array}{c}24 / \\
25\end{array}$ & 96 & 100 & 100 & 96 & $\begin{array}{l}22 / \\
25\end{array}$ & 89 & 100 & 100 & 88 & 8 to. 32 \\
\hline $\mathrm{D}$ & $\begin{array}{c}34 / \\
35\end{array}$ & 97 & 100 & 100 & 93 & $\begin{array}{c}33 / \\
35\end{array}$ & 94 & 100 & 100 & 87 & 8 to. 32 \\
\hline IMP & $\begin{array}{c}20 / \\
20\end{array}$ & 100 & 100 & 100 & 100 & $\begin{array}{c}20 / \\
20\end{array}$ & 100 & 100 & 100 & 100 & 8 to. 32 \\
\hline VIM & $\begin{array}{c}19 / \\
19\end{array}$ & 100 & 100 & 100 & 100 & $\begin{array}{c}19 / \\
19\end{array}$ & 100 & 100 & 100 & 100 & 8 to. 32 \\
\hline SIM & $8 / 8$ & 100 & 100 & 100 & 100 & $8 / 8$ & 100 & 100 & 100 & 100 & 8 to. 32 \\
\hline SPM & $\begin{array}{c}17 / \\
17\end{array}$ & 100 & 100 & 100 & 100 & $\begin{array}{c}17 / \\
17\end{array}$ & 100 & 100 & 100 & 100 & 8 to. 32 \\
\hline GIM & $21 / 21$ & 100 & 100 & 100 & 100 & $21 / 21$ & 100 & 100 & 100 & 100 & 8 to. 32 \\
\hline
\end{tabular}

${ }^{*} \mathrm{NPV}$ : negative predictive values. ${ }^{*} \mathrm{PPV}$ : positive predictive values.

TABle 6: Results of sensitivity, specificity, NPV, and PPV in MHT versus IMP.EDTA test.

\begin{tabular}{|c|c|c|c|c|c|c|c|c|c|c|}
\hline & \multirow{2}{*}{ No. } & \multicolumn{4}{|c|}{ MHT } & \multirow{2}{*}{ No. } & \multicolumn{4}{|c|}{ IMP.EDTA } \\
\hline & & Sensitivity (\%) & Specificity (\%) & $\mathrm{PPV}^{*}(\%)$ & $\mathrm{NPV}^{*}(\%)$ & & Sensitivity (\%) & Specificity (\%) & PPV (\%) & NPV (\%) \\
\hline A & $5 / 11$ & 64 & 95 & 84 & 86 & $6 / 11$ & 68 & 88 & 68 & 88 \\
\hline B & $23 / 40$ & 70 & 97 & 97 & 34 & $22 / 40$ & 68 & 90 & 97 & 33 \\
\hline $\mathrm{C}$ & $14 / 25$ & 69 & 96 & 96 & 68 & $16 / 25$ & 73 & 88 & 89 & 72 \\
\hline $\mathrm{D}$ & $18 / 35$ & 67 & 93 & 97 & 45 & $20 / 35$ & 70 & 87 & 94 & 48 \\
\hline IMP & $15 / 20$ & 80 & 93 & 90 & 85 & $13 / 20$ & 74 & 87 & 83 & 80 \\
\hline VIM & $14 / 19$ & 79 & 93 & 90 & 85 & $10 / 19$ & 67 & 88 & 82 & 76 \\
\hline SIM & $4 / 8$ & 66 & 95 & 80 & 91 & $3 / 8$ & 61 & 87 & 57 & 89 \\
\hline SPM & $11 / 17$ & 73 & 93 & 89 & 84 & $10 / 17$ & 70 & 88 & 80 & 82 \\
\hline GIM & $11 / 21$ & 67 & 93 & 91 & 73 & $12 / 21$ & 70 & 87 & 84 & 75 \\
\hline
\end{tabular}

${ }^{*} \mathrm{NPV}$ : negative predictive values. ${ }^{*} \mathrm{PPV}$ : positive predictive value.

carbapenemase-producing isolates. The MHT test is a simple test that is performed to detect carbapenemase-producing isolates. Some studies have revealed that the MHT test has false-negative and false-positive results [23]. In the current study, the sensitivity and NPV of the MHT test for the detection of MBL producers, with 17 missing detections, were $(23 / 40,70 \%)$ and $34 \%$, respectively. Moreover, the MHT test had $(18 / 35,67 \%)$ sensitivity and $45 \%$ NPV for the detection of class D (OXA-48) carbapenemase. The results of Pasteran et al. study showed that the MHT test had low sensitivity (78\%) and specificity (57\%) for the detection of MBL and KPC-producing $P$. aeruginosa isolates [24]. Carvalhaes in 2010 reported $93 \%$ sensitivity for the MHT test and showed the possibility of false-positive results in the MHT test [25]. These false-positive results might be due to the porin loss in the cell wall of bacteria [26]. Furthermore, the lowest NPV value was related to MBL production, so this test is not a suitable method for the identification of MBLproducing isolates. In our study, as Varaiya et al. [27] and Murugan et al. [28] studies, the sensitivity of CarbaNP and CIM tests for the detection of group B carbapenemase was $100 \%$. Among the rapid test which is used to identify MBLproducing $P$. aeruginosa isolates, the selection of simple screening tests like the CDDT test is a critical stage to the monitoring of the emerging resistant determinants. In a study, $147 \mathrm{P}$. aeruginosa isolates had MBL genes, and the CDDT test with high sensitivity as a rapid test for class B ambler was introduced [29]. Since the prevalence of the IMP gene in carbapenem-resistant isolates was high, this test can be used in the early screening of these resistant isolates. Due to the low NPV value and sensitivity of the MHT test, this method is not recommended for the identification of carbapenemase-producing isolates.

CDDT test was used for the detection of KPC, MBL, AmpC, and OXA-48 genes, with 68\%, 68\%, 73\%, and 70\% sensitivity, respectively. The lowest sensitivity of the CDDT test was related to the detection of MBL, so it is not recommended for the detection of class B carbapenemaseproducing isolates.

The CDDT could be very helpful in daily works in the laboratories to achieve rapid detection among $P$. aeruginosa isolates producing KPC and MBL carbapenemase enzymes. The NPV results of the CDDT test for the detection of MBL producers were as low as the MHT test.

The performance of the CIM and CarbaNP tests for the identification of MBL, KPC, AmpC, and OXA-48 gene 
producers was also evaluated. The overall sensitivity, specificity, PPV, and NPV of the CIM test for KPC-producing isolates were $100 \%$.

In the present study, the sensitivity of the CIM test for the detection of MBL, OXA-48, and AmpC genes was 97\%, $94 \%$, and $89 \%$, respectively. Moreover, the specificity of the CIM test for the detection of MBL, OXA-48, and AmpC genes was $100 \%$. In a study by Elif Aktaș et al., the sensitivity and specificity of the CIM test were $78 \%$ and $100 \%$, respectively. Elif Aktaş et al. also showed that there was no significant difference in incubation times [30]. Therefore, the CIM test can be a good alternative test for the identification of carbapenemase-producing isolates [31].

In addition to CIM, the CarbaNP test was recommended for epidemiological or infection control purposes by the CLSI and has wide applications due to the high sensitivity and specificity [7]. However, some disadvantages of this method for the detection of carbapenemase-producing isolates are not just the high cost for the preparation of imipenem powder, but also the low sensitivity of this method for the detection of the OXA-type carbapenemases, especially the OXA-48 positive strains [7]. It has been shown that the protein extraction buffer used in the CarbaNP prevented the color change in the reaction known as the buffer effect, which was the leading cause in the identification of the OXA producers.

The CIM test was first recommended by CLSI in 2017. There are some advantages of this test, including low cost, high sensitivity and specificity, and ease of interpretation of results that make it a suitable phenotypic method for the identification of carbapenemase-producing isolates.

According to the results of this study, among the four phenotypic methods, the CarbaNP and the CIM methods can be used for rapid and appropriate detection of carbapenemase-producing isolates to control the infection and prevent the prevalence of these isolates. It can also be used in routine clinical microbiological laboratories.

\section{Conclusion}

The results of this study showed that the CarbaNP and CIM tests have high sensitivity and specificity for identification of carbapenemase-producing $P$. aeruginosa isolates. Due to their ease of use and the rapidity of these methods, these can be used as very suitable methods for the detection of carbapenemase-producing $P$. aeruginosa isolates in clinical laboratories and medical diagnosis.

\section{Abbreviations}

MHT: Modified Hodge test

CDDT: Combined double-disk synergy test

CIM: Carbapenem inactivation method

MIC: Minimal inhibitory concentration

PCR: Polymerase chain reaction

SIM: $\quad$ Sulfide indole motility

TSI: Triple sugar iron agar

BHI: $\quad$ Brain heart infusion media

EDTA: Ethylenediaminetetraacetic acid

MHA: Muller-Hinton Agar.

\section{Data Availability}

The data and material used are available upon request.

\section{Ethical Approval}

This study was approved by the ethics committee of Hamadan University of Medical Sciences (Code No.: IR.UMSHA.REC.1396.662).

\section{Consent}

Written informed consent was taken from all participants.

\section{Conflicts of Interest}

The authors declare that there are no conflicts of interest.

\section{Authors' Contributions}

MB performed the tests and collected and analyzed the data. MT was a contributor in writing and editing the manuscript. MRA designed the project and contributed in the whole steps of the project. All the authors read and approved the final manuscript.

\section{Acknowledgments}

The authors of this article are grateful to Hamadan University of Medical Sciences for their financial support in conducting the research. This work was supported by the Vice Chancellor and Research Technology of Hamadan University of Medical Sciences on Grant no. 9610126514. This work was supported by the Vice Chancellor and Research Technology of Hamadan University of Medical Sciences.

\section{References}

[1] J. L. Murray, T. Kwon, E. M. Marcotte, and M. Whiteley, "Intrinsic antimicrobial resistance determinants in the superbug Pseudomonas aeruginosa," mBio, vol. 6, no. 6, Article ID e01603, 2015.

[2] Y. Carmeli, J. Armstrong, P. J. Laud et al., "Ceftazidimeavibactam or best available therapy in patients with ceftazidime-resistant Enterobacteriaceae and Pseudomonas aeruginosa complicated urinary tract infections or complicated intra-abdominal infections (REPRISE): a randomised, pathogen-directed, phase 3 study," The Lancet: Infectious Diseases, vol. 16, no. 6, pp. 661-673, 2016.

[3] Z. Pang, R. Raudonis, B. R. Glick et al., “Antibiotic resistance in Pseudomonas aeruginosa: mechanisms and alternative therapeutic strategie," Biotechnology Advances, vol. 37, no. 1, pp. 177-192, 2018.

[4] K. Bush, "The importance of $\beta$-lactamases to the development of new $\beta$-lactams," Antimicrobial Drug Resistance, vol. 21, no. 6, pp. $165-175,2017$.

[5] E. A. Idelevich, K. Sparbier, M. Kostrzewa, and K. Becker, "Rapid detection of antibiotic resistance by MALDI-TOF mass spectrometry using a novel direct-on-target microdroplet growth assay," Clinical Microbiology and Infection, vol. 24, no. 7, pp. 738-743, 2018. 
[6] B. Bayraktar, A. Barıș, G. Malkoçoğlu, D. Erdemir, and N. Kına, "Comparison of carba NP-direct, carbapenem inactivation method, and $\beta$-CARBA tests for detection of carbapenemase production in enterobacteriaceae," Microbial Drug Resistance, vol. 25, no. 1, pp. 97-102, 2019.

[7] K. Sun, X. Xu, J. Yan, and L. Zhang, "Evaluation of six phenotypic methods for the detection of carbapenemases in gram-negative bacteria with characterized resistance mechanisms," Annals of Laboratory Medicine, vol. 37, no. 4, pp. 305-312, 2017.

[8] A. A. Galvani and A. Tukmechi, "Determination of the prevalence of metallo- $\beta$-lactamases producing Pseudomonas aeruginosa strains from clinical samples by imipenem-EDTA combination disk method in Mottahari and Emam Khomaini hospitals of Urmia," Public Health Reports, vol. 1, no. 2, pp. 5-58, 2015.

[9] Clinical and Laboratory Standards Institute, Performance Standards for Antimicrobial Susceptibility Testing (CLSI Supplement M100), Clinical and Laboratory Standards Institute, Wayne, PA, USA, 30th edition, 2020.

[10] R. Sujatha, R. Goyal, and V. Mishra, "Detection of metallo beta lactamase producing Pseudomonas aeruginosa among clinical isolates," Australas Medical Journal, vol. 6, no. 11, pp. 686-693, 2017.

[11] S. Datta, R. Dey, J. Dey, and S. Ghosh, "A comparative study of modified Hodge test and Carba NP test for detecting carbapenemase production in Gram-negative bacteria," Medical Journal of Dr. D.Y. Patil University, vol. 10, no. 4, pp. 365-369, 2017.

[12] R. Bir, S. Mohapatra, A. Kumar et al., "Comparative evaluation of in-house Carba NP test with other phenotypic tests for rapid detection of carbapenem-resistant Enterobacteriaceae," Journal of Clinical Laboratory Analysis, vol. 33, no. 1, Article ID e22652, 2019.

[13] P. J. Simner, J. K. Johnson, W. B. Brasso et al., "Multicenter evaluation of the modified carbapenem inactivation method and the carba NP for detection of carbapenemase-producing Pseudomonas aeruginosa and acinetobacter baumannii," Journal of Clinical Microbiology, vol. 56, no. 1, 2018.

[14] A. Crowe, L. Brenton, M. Kingston, D. Jardine, and M. J. Waters, "Comparison of the carbapenem inactivation method (CIM) and modified carbapenem inactivation method (mCIM) for the detection of carbapenemase-producing organisms," Pathology, vol. 50, no. 7, pp. 764-766, 2018.

[15] P. L. Ho, Y. Wang, C. W. S. Tse et al., "Rapid detection of carbapenemase production in Enterobacteriaceae by use of a modified paper strip Carba NP method," Journal of Clinical Microbiology, vol. 56, no. 1, 2017.

[16] O. B. Ahmed, A. H. Asghar, and M. Elhassan, "Comparison of three DNA extraction methods for polymerase chain reaction (PCR) analysis of bacterial genomic DNA," African Journal of Microbioliology Research, vol. 8, no. 6, pp. 598-602, 2014.

[17] M. Beig, M. Taheri, and M. R. Arabestani, "Expression of MexAB-OprM efflux pump and OprD porin in carbapenemase producing Pseudomonas aeruginosa clinical isolates," Gene Reports, vol. 20, no. 2, pp. 1-8, 2020.

[18] T.-H. Wang, Y.-S. Leu, N.-Y. Wang, C.-P. Liu, and T.-R. Yan, "Prevalence of different carbapenemase genes among carbapenem-resistant Acinetobacter baumannii blood isolates in Taiwan," Antimicrobial Resistance \& Infection Control, vol. 7, no. 1, p. 123, 2018.

[19] D. Paul, D. Chanda, A. P. Maurya et al., "Co-carriage of blaKPC-2 and blaNDM-1 in clinical isolates of Pseudomonas aeruginosa associated with hospital infections from India," PLoS One, vol. 10, no. 12, Article ID e0145823, 2015.

[20] J.-L. Dumas, C. Delden, K. Perron, and T. Köhler, "Analysis of antibiotic resistance gene expression in Pseudomonas aeruginosa by quantitative real-time-PCR," FEMS Microbiology Letters, vol. 254, no. 2, pp. 217-225, 2006.

[21] M. F. Mushi, S. E. Mshana, C. Imirzalioglu, and F. Bwanga, "Carbapenemase genes among multidrug resistant gram negative clinical isolates from a tertiary hospital in Mwanza, Tanzania," BioMed Research International, vol. 2014, Article ID 303104, 6 pages, 2014.

[22] S. R. Evans, A. M. Hujer, H. Jiang et al., "Informing antibiotic treatment decisions: evaluating rapid molecular diagnostics to identify susceptibility and resistance to carbapenems against acinetobacter spp. in PRIMERS III," Journal of Clinical Microbiology, vol. 55, no. 1, pp. 134-144, 2017.

[23] P. Wang, S. Chen, Y. Guo et al., "Occurrence of false positive results for the detection of carbapenemases in carbapenemase-negative Escherichia coli and Klebsiella pneumoniae isolates," PLoS One, vol. 6, no. 10, Article ID e26356, 2011.

[24] F. Pasteran, O. Veliz, D. Faccone et al., "A simple test for the detection of KPC and metallo- $\beta$-lactamase carbapenemaseproducing Pseudomonas aeruginosa isolates with the use of meropenem disks supplemented with aminophenylboronic acid, dipicolinic acid and cloxacillin," Clinical Microbioliology and Infection, vol. 17, no. 9, pp. 1438-1441, 2011.

[25] C. G. Carvalhaes, R. C. Picao, A. G. Nicoletti, D. E. Xavier, and A. C. Gales, "Cloverleaf test (modified Hodge test) for detecting carbapenemase production in Klebsiella pneumoniae: be aware of false positive results," Journal of Antimicrobial Chemotherapy, vol. 65, no. 2, pp. 249-251, 2010.

[26] M. Doumith, M. J. Ellington, D. M. Livermore, and N. Woodford, "Molecular mechanisms disrupting porin expression in ertapenem-resistant Klebsiella and Enterobacter spp. clinical isolates from the UK," Journal of Antimicrobial Chemotherapy, vol. 63, no. 4, pp. 659-667, 2009.

[27] A. Varaiya, M. Kulkarni, P. Bhalekar, and J. Dogra, "Incidence of metallo-beta-lactamase-producing Pseudomonas aeruginosa in diabetes and cancer patients," Indian Journal of $\mathrm{Pa}$ thology and Microbiology, vol. 51, no. 2, p. 200, 2008.

[28] S. Murugan, R. B. Lakshmi, D. Uma et al., "Prevalence and antimicrobial susceptibility pattern of metallo $\beta$ lactamase producing Pseudomonas aeruginosa in diabetic foot infection," Interanational Journal Microbiology Research, vol. 1, no. 3, pp. 123-128, 2010.

[29] R. Sachdeva, B. Sharma, and R. Sharma, "Evaluation of different phenotypic tests for detection of metallo- $\beta$-lactamases in imipenem-resistant Pseudomonas aeruginosa," Journal of Laboratory Physicians, vol. 9, no. 4, pp. 249-253, 2017.

[30] E. Aktaş, G. Malkoçoğlu, B. Otlu et al., "Evaluation of the carbapenem inactivation method for detection of carbapenemase-producing gram-negative bacteria in comparison with the RAPIDEC CARBA NP," Microbial Drug Resistance (Larchmont, N.Y.), vol. 23, no. 4, pp. 457-461, 2017.

[31] P. Nordmann, M. Gniadkowski, C. G. Giske, L. Poirel, N. Miriagou, and V. Miragou, "Identification and screening of carbapenemase-producing Enterobacteriaceae," Clinical Microbiology and Infection, vol. 18, no. 5, pp. 432-438, 2012. 